\title{
Study on the Teaching of Internet + Era Virtual Packaging Design Ying Luo ${ }^{1}$ \\ ${ }^{1}$ WUHAN POLYTECHNIC, Wuhan 430000, China
}

\author{
Keywords: Internet; Era; Virtual Packaging Design; Teaching
}

\begin{abstract}
With the continuous development of the information technology, the level of the Internet technology is becoming higher and higher, and the Internet has already become an important content in people' s life and work. Subsequently, the virtual technology is constantly improving, and virtual products have also emerged. Thus, in this era, virtual packaging design is a major important trend at present and in the future. In teaching, colleges and universities should attach great importance to the Internet + era virtual packaging design and scientifically design relevant courses to make students have an in-depth understanding of the Internet + era virtual packaging design and mater corresponding design knowledge.
\end{abstract}

\section{Introduction}

In the history of human civilization in the world, when products appeared, packaging was born. The features of the era can be fully reflected through packaging. Meanwhile, with the continuous development of the era, the packaging design is also constantly developing. In recent years, the computer information technology is developing faster and faster, ad virtual reality technology is also becoming more and more perfect. In people' s daily life, the Internet is producing a greater and greater impact. Thus, people' s living space and production space are also further expanded, giving birth to a new space, i.e., virtual space. Subsequently, a new product is produced, i.e., virtual product. In the packaging design industry, the design of virtual product packaging is a major research topic both at present and in the future. Therefore, in teaching, each college and university should attach great importance to the design of the Internet + era virtual packaging and scientifically design relevant courses to make students have an in-depth understanding of the design of the Internet + era virtual packaging design and master relevant design knowledge. Therefore, this paper is of great significance to the in-depth research into the teaching performance of the Internet + era virtual packaging design.

\section{Key Contents of Teaching Virtual Packaging Design}

\section{It Should Comply with the Ergonomic Principles}

The design of the virtual packaging should be consistent with the principles of ergonomics. In general, while purchasing furniture, people often prefer the rounded corner furniture, and few people prefer furniture with sharp corners because most people believe that if an object is too sharp, it can easily scratch people, so most people prefer the more rounded objects. Although people may not be able to articulate the reason why they prefer rounded objects. In fact, from the perspective of science, rounded objects are more in line with the ergonomic principles. Although virtual sharp objects may not scratch people, people have already formed this psychological habit. Therefore, when designing the virtual packaging model, in-depth reflection must be given to people' $\mathrm{s}$ psychological comfort to fully reflect the people-oriented principle and make the virtual packaging design beautiful and eye-catching.

\section{Follow the Nature}

In the design of virtual packaging modeling, an in-depth study and understanding of nature is necessary. It is necessary to apply bionics, imitate the form of a natural object and clarify the important integration between nature and artificial things to make it easy for virtual packaging model through clever handling of art. In this way, the virtual packaging will have a natural beauty, produce resonance with users in terms of emotion and fully stimulate users' tactile experience. 


\section{Key Contents of Teaching the Selection of Virtual Packaging Materials}

\section{The Selected Materials Should Have Special Texture Beauty}

The texture of the material, which is expressed by the visual sense and the tactile sense, is actually a complex of different external characteristics. The real material can be moderated and reproduced with the simulation of the virtual packaging material. With the continuous development and application of artificial materials and natural materials, the virtual packaging can carry out more different forms of simulation and can show more texture. Currently, there are so many different types of materials that modern designs can use, and there is a big difference between the textures they show for different types of materials. For a variety of texture materials, people can have different psychological feelings. In the virtual packaging design, it is a very crucial part as which kind of material should be chosen and which texture should be fully reflected. The rational application of materials, full application of materials' advantages and effective avoidance of materials' shortcoming can effectively improve the materials' functions and highlight the quality of materials.

\section{Attach Great Importance to the Tactile Design of Virtual Packaging}

In the process of designing the virtual packaging, in order to effectively improve the quality of the virtual packaging and effectively guide the operation of the designer, tactile design should be carried out. Tactile affinity is strongest among all kinds of perceptions, and there is a close relationship between tactile presentation and selection of materials. In the choice of virtual packaging materials, we must take full account of the natural properties of the material so as to produce a very affinity effect. Whether a design is attentive in design and whether the design works can impress customers mainly depend on the details of the design work. In tactile design of virtual packaging, importance must be attached to the in-depth mining of emotions to impress customers in a relatively natural way.

\section{Key Contents of Teaching Virtual Packaging Graphic Image Design}

\section{Graphic Design}

\section{Properly Deal with the Relationship between Points, Lines and Surfaces}

The visual effect is the result of the virtual packaging design, but whichever kind of visual effect is the graphic formed by the reasonable combination of the three elements of point, line and surface. In order to design high-quality virtual packaging graphics with a very high sense of visual sense and order, designers must carefully arrange the three elements of point, lines and surface and strictly comply with the rules of different forms, such as radiation, symmetry, rhythm and so on. In order to fully show the mood of the images and the best virtual effect, it is necessary to properly deal with the relationship between points, lines and surface.

\section{Properly Deal with the Relationship between Abstract and Figurative Things}

In the design of virtual packaging graphics, it is necessary to ensure that the reality and virtuality can complement with each other. At the real position of the image, specific and intuitive graphics must be designed so as to make users have a more complete and profound impression. At the virtual position of the graphics, it is necessary to design abstract graphics so as to enrich the graphics contents and make people produce associations. A perfect combination of abstract and figurative things can more quickly convey the theme of virtual packaging design.

\section{Properly Deal with the Relationship between Network Band width and the Image Effect}

At present, in the process of obtaining network information, the network bandwidth will still have a certain constraint on them. Among them, the important factor that people gain the speed of network information is the size of the graph. Therefore, the design of the virtual packaging, the graphics used must have a very good visual effect and small size. Under the same conditions of expression, in order to effectively save the limited network bandwidth resources, vector graphics should be used as far as possible so as to pass on the virtual packaging information smoothly. 


\section{Text Design}

\section{The Principle of Readability of Texts Should be Followed}

When obtaining the text information, people often pay attention to the most eye-catching part of the content first, and then quickly browse and search this part of the content, so in the design of the virtual packaging text, we must ensure that the text is readable. First, Aiming at text size and illegible text fonts that exceed the visual comfort of consumers, we should avoid them in the design of virtual packaging, and then use the font size and fonts as the main basis for scientifically combining and aligning text alignment and spacing so as to effectively improve the legibility of textual content; second, The importance of the textural contents should be taken as the main basis for the rational layout of different fonts and text font sizes, and customers should be effectively guided when customers select and identify the texts; third, the fonts in most customers' computers are naturally equipped in the system, so it is better to select thee default fonts as far as possible. When there are special needs, the uncommonly used fonts should be converted so that all the computer terminals can accurately acquire the text information; fourth, choose the reasonable text colors according to the background color to make them maintain a coordinated relationship.

\section{The Principle of Accuracy of Words Should be Followed}

For the attributes of the virtual package, the text can authoritatively describe it. Therefore, the text content in the virtual package must be able to fully reflect the theme of the package, accurately convey the information of the virtual product, and no ambiguity is allowed. In the meantime, more wording should be applied to make the user feel more psychologically based on the correctness of the written meanings.

\section{Color Design}

In the design of the color of the virtual packaging, the designer must have a deep understanding of the product features, the market positioning of the product and the characteristics of the enterprise. Through scientific analysis, the color of the virtual packaging design should be guaranteed to be specific. People' $s$ inner feeling of colors and the scientific rule of color should be taken as the main basis to reconcile, compare and apply colors so as to make the colors in virtual packaging effectively convey the product information. It is noteworthy that in different displays, even the colors of the same RGB value can have different display effects, which is mainly caused by the browser, display card and display, etc. In order to effectively solve the problem of color deviation, it is necessary to make use of the color safety of web pages.

\section{Key Contents of Teaching Virtual Packaging Sound Design}

\section{Design of Operation Prompt Sound}

In the virtual package, the main characteristics of a single action sound prompts include jump sense, single tone and slow tempo, but the main features of bulk information processing tone include fast-paced appearance and continuous tone. In the design of virtual packaging operation beeps, in order to avoid the emergence of emerging recognition barriers caused by the use of the value of the sound is still lost, be sure to clearly distinguish between and background music. In addition, for all operation beacons in the same virtual packaging system, the principle of consistency should be obeyed, and the common design habit of people should be based on the common meaning of sound and the design of the operation beeps on different occasions to achieve standardization. Through visual guidance and the pursuit of positive operation prompt sound, effectively establish the joint feedback mechanism and fully play the independent and interactive ability of visual sense in some special cases so as to effectively improve users' memory and adaptiveness and operation efficiency.

\section{Sound Effect Design}

While designing the music for virtual packaging, the task to be completed is to imply the action and effectively simulate the sound of actual actions. Herein, for the content of the task completed above, it is often used when the user's attention is drawn to lead other topics. In the design of sound effects, the sound of various actions must be highly simulated and modestly exaggerated. Even in 
real life, some actions will not produce sound, but in order to effectively prompt the information, the atmosphere is effectively rendered in the virtual packaging design, and these sounds should also be related to the sound motion design.

\section{Voice Design}

The dialogue between people and the computer is the main content of virtual packaging voice design. Voice can only be used in important contents so as to serve as tips and explanation and provide a more user-friendly interface for users. Listening comfortably with full tone and clear sound quality is the main purpose of virtual packaging voice design. While designing virtual packaging voice, theme demands should be taken as the main basis, ad dubbing personnel should reasonably select the voice quality so as to fully reflect the emotional atmosphere. For example, a man' s voice with a sense of vicissitude should be given when opening a treasure box that has been sealed for a long time with a prompt so as to bring out the atmosphere; and a woman' soft voice should be given when opening a virtual packaging of soft style.

\section{References}

[1] Man Nie: Art Sea,Vol. 96 (2017) No.9, p.12-13.

[2] Jia Liu: Art Education, Vol. 121(2017) No.11, p.15-16.

[3] Yan Li: Theater House, Vol. 56(2017) No.9, p.44-45.

[4] Xiangyang Guo: Chinese and Foreign Entrepreneurs, Vol. 68(2017) No.14, p.26-27.

[5] Weiying Lin: Art World, Vol. 108(2017) No.7, p.31-32.

[6] Fei Liu: Educational Innovation, Vol. 114 (2017) No.7, p.87-88.

[7] Huawen Xie: Decoration, Vol. 43(2017) No.3, p.8-9.

[8] Huiting Chen: Art Observation, Vol. 136(2017) No.5, p.43-44.

[9] Wei Jie: Graphically creative (China building industry press, 2013), p. 38-49.

[10] Donald a. Norman : design psychology (China citic publishing house, 2016), p. 86-102. 